\title{
In-plane remote photoluminescence excitation of carbon nanotube by propagating surface plasmon
}

\author{
Padmnabh Rai, ${ }^{1}$ Nicolai Hartmann, ${ }^{2}$ Johann Berthelot, ${ }^{1,3}$ Gérard Colas-des-Francs,,${ }^{1}$ \\ Achim Hartschuh, ${ }^{2}$ and Alexandre Bouhelier ${ }^{1, *}$ \\ ${ }^{1}$ Laboratoire Interdisciplinaire Carnot de Bourgogne, CNRS UMR 6303, Université de Bourgogne, Dijon 21078, France \\ ${ }^{2}$ Department Chemie and CeNS, Ludwig-Maximilians-Universität München, München 81377, Germany \\ ${ }^{3}$ The Institut de Ciències Fotòniques (ICFO), Av. Carl friedrich Gauss, Castelldefels 08860, Spain \\ *Corresponding author: alexandre.bouhelier@u-bourgogne.fr
}

Received July 18, 2012; revised September 13, 2012; accepted September 25, 2012;

posted September 25, 2012 (Doc. ID 172562); published November 12, 2012

In this work, we demonstrate propagating surface plasmon polariton (SPP) coupled photoluminescence (PL) excitation of single-walled carbon nanotube (SWNT). SPPs were launched at a few micrometers from individually marked SWNT, and plasmon-coupled PL was recorded to determine the efficiency of this remote in-plane addressing scheme. The efficiency depends upon the following factors: (i) longitudinal and transverse distances between the SPP launching site and the location of the SWNT and (ii) orientation of the SWNT with respect to the plasmon propagation wave vector $\left(\boldsymbol{k}_{\mathrm{SPP}}\right)$. Our experiment explores the possible integration of carbon nanotubes as a plasmon sensor in plasmonic and nanophotonic devices. (c) 2012 Optical Society of America

OCIS codes: $\quad 240.6680,300.2530,120.0280$.

The superior electrical transport and the unique optical properties of single-walled carbon nanotubes (SWNTs) have become a center of interest for two reasons. One, from the viewpoint of nanoscale physics and their applications in the next generation nano-optoelectronic devices $[\underline{1}, \underline{2}]$ ranging from photon generation $[3,4]$, and from nanoscale integrated photonic detectors $[\overline{5}]$. Combined with plasmonics, these one-dimensional systems offer a potential technological solution to interface near-field optics and nano-electronics in the pursuit to establish a controlled optical exchange. A device, based upon such a platform, requires increasing the knowledge of the physical laws governing communication and exchange mechanisms between functional elements on nanometer length scales. For instance, electromagnetic near-field interactions between individual quantum objects and propagating surface plasmons needs to be understood with respect to fundamental parameters governing the interfacing mechanisms. Attempts were made in [ㅌ, 7$]$ by addressing semiconducting quantum dots via running a surface plasmon. Recently, Hartmann et al., reported the launching of surface plasmon polaritons (SPPs) in metal films by single SWNT acting as dipolar emitters, indicating an energy transfer from the nanotube excitons to the surface plasmons [8]. The demonstration of enhanced spontaneous emission of semiconducting materials near nanostructured metallic surfaces [9], exciton-plasmon conversion $[\underline{8}, \underline{10}, \underline{11}]$, and large optical gain [12] create a strong motivation to explore the integration of SWNT optical properties in a plasmonic platform.

In this Letter, we report on remote in-plane photoluminescence (PL) excitation of individual SWNT by propagating SPPs in metal thin film strip waveguides. Our experiment provides critical information on the coupling properties between a propagating surface plasmon mode and electronic resonances of an individual SWNT.

High pressure carbon monoxide (HiPCO) synthesized SWNTs were dispersed in a $1 \%$ wt. sodium dodecyl sulfate solution by sonication and were subsequently processed by centrifugation [8]. The nanotubes used in our experiment range in diameter between 0.7-1.2 nm. Nanotubes were randomly deposited on top of plasmonic waveguides by spin coating. The strip waveguides were fabricated by standard electron-beam lithography and lift-off processes on indium tin oxide (ITO)-coated cover glass slips. The structure consists of Au strips of varying length $(\sim 10-30 \mu \mathrm{m})$, with a width of $5 \mu \mathrm{m}$ and a thickness of $35 \mathrm{~nm}$. On top of the Au waveguides, an additional spacer consisting of a $10 \mathrm{~nm}$ thick $\mathrm{SiO}_{x}(x=1.5-2.0)$ layer was deposited by thermal evaporation to prevent quenching of the nanotube's PL via coupling to largemomentum "lossy" modes in the metal film that decay nonradiatively [13]. We used an inverted optical microscope with an oil immersion objective $(\mathrm{NA}=1.49)$ and a $p$-polarized laser emitting at $\lambda=785 \mathrm{~nm}$ for launching SPPs along the waveguide in a Kretschmann-like configuration. Leakage radiation microscopy was used to determine the characteristics of the SPPs [14]. Our choice of wavelength is motivated by the relatively long propagation length of SPPs in the near-infrared spectral region. Although, the excitation efficiency of nanotubes, at this wavelength not matching $E_{22}$ energies is reduced. However, their excitation is still possible through phonon-assisted transitions [15].

SPPs were launched at a few micrometer from individually marked SWNT. Their PL emission was recorded by a charge-coupled device (CCD) camera positioned at a conjugated image plane of the microscope. A combination of cutoff filters (dichroic at $785 \mathrm{~nm}$, and longpass at $850 \mathrm{~nm}$ ) was used to suppress direct laser transmission and to select the emission of $(9,1),(8,3)$, and $(6,5)$ nanotubes.

A scanning electron micrograph (SEM) of individual SWNT placed on a plasmonic waveguide is shown in Fig. 1(a). The launching of propagating SPPs in the strip waveguide is illustrated in the leakage radiation image of Fig. 1(b). The plasmon is excited in the Au reservoir and propagates from left to right as indicated by the propagation wave vector $\left(\boldsymbol{k}_{\mathrm{SPP}}\right)$. The image was acquired without 


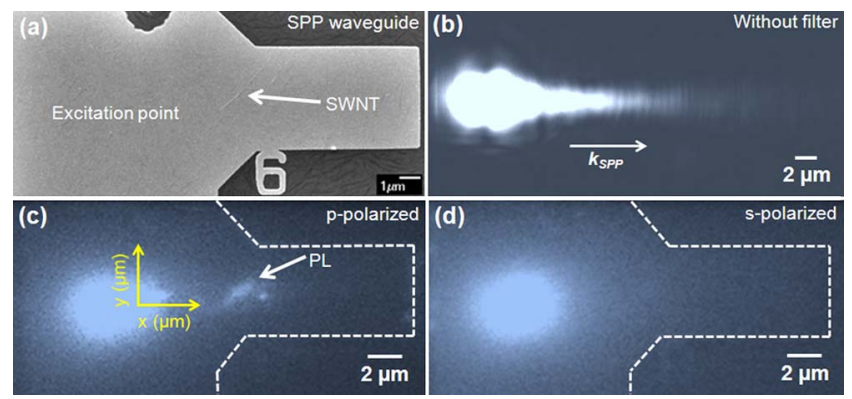

Fig. 1. (Color online) (a) SEM image of an individual SWNT located on an Au strip waveguide, (b) leakage radiation image of SPP propagating along the Au strip waveguide, (c) SPPcoupled PL from the SWNT, and (d) cross-check experiment for $s$-polarized excitation.

any spectral filtering. Figure $1(\mathrm{c})$ was obtained with spectral filtering and an acquisition time of 120 seconds for a laser excitation intensity $13 \mathrm{~kW} / \mathrm{cm}^{2}$. PL emission of the nanotube, indicated by an arrow in Fig. 1(a), is observed at a distance of approximately $\sim 4 \mu \mathrm{m}$ from the laser beam suggesting a remote excitation of the nanotube by the SPPs. Residual laser light not completely suppressed by the cutoff filters is visible at the launching site due to a small angular tilt of the excitation beam on the rejection interference filter. To confirm the SPP-induced PL excitation, we rotated the polarization of the incoming laser light by $90^{\circ}$. For this $s$-polarization SPPs are no longer excited and consequently no light emission at the location of nanotube is observed [Fig. 1(d)]. Furthermore, we found that the PL emission was inhibited after subsequent SEM analysis. Exposure of SWNTs to an electron beam is known to degrade its PL properties [16]. This strongly supports the PL origin of light recorded at the position of the nanotube in Fig. 1(c).

Theoretical and experimental studies clarified that optical transitions in SWNTs are dominated by exciton dyanamics where excited electrons and holes are strongly confined to form a bound exciton state of energy $(\sim 0.3-1.0 \mathrm{eV})$ in small diameter semiconducting nanotubes [17-19]. The excitation efficiency of plasmonexciton interaction in semiconducting SWNT is determined here by studying the intensity variation of plasmon-mediated PL with respect to the longitudinal and transverse profiles of the propagating SPP mode. Longitudinal ( $x$-axis) and transverse ( $y$-axis) distances between the SPP excitation spot and the coupled PL of the nanotube are represented by the reference frame $(x-y)$ in Fig. 1(c). The SPP mode profiles were extracted from the direct leakage radiation image [Fig. 1(b)]. The distance between the position of the nanotube and the excitation spot was controlled by piezo-actuators. The background corrected SWNT PL intensity is plotted against varying longitudinal distances from the SPP excitation spot in Fig. 2(a). The PL intensity decreases exponentially with the increased longitudinal distance from the SPP excitation point. To investigate the longitudinal coupling efficiency, we compared the intensity of the PL response with the propagating SPP profile. The longitudinal intensity variation of the SPP mode is given by the expression $I_{L} \approx I_{0} \exp \left(-x / L_{\mathrm{SPP}}\right)$, where $x$ is the longitudinal variable and $L_{\mathrm{SPP}}$ is the decay length of SPP. $L_{\mathrm{SPP}}$ depends on the dielectric constants of $\mathrm{Au}$
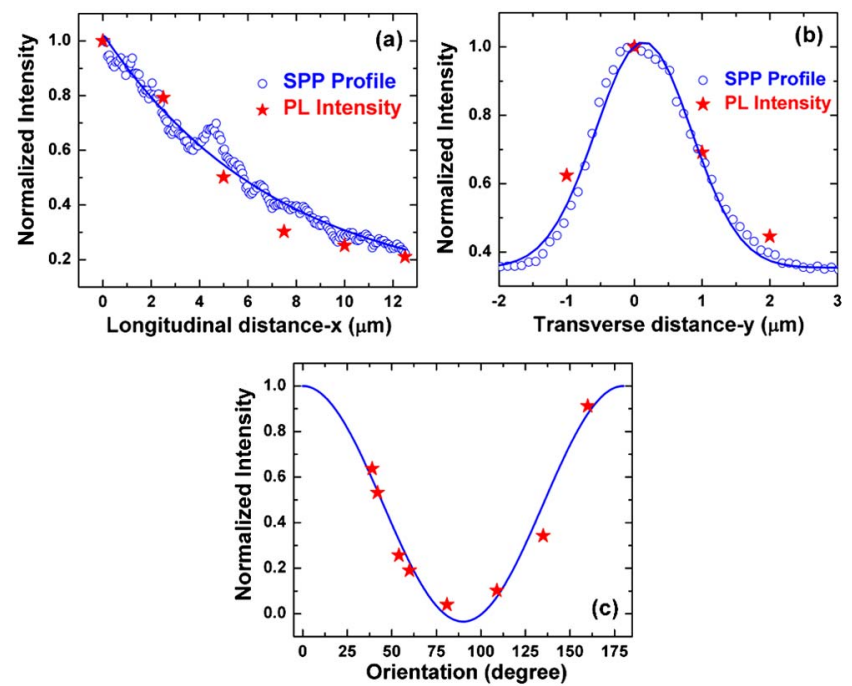

Fig. 2. (Color online) (a) and (b) are normalized intensity plot of SPP profile and plasmon-coupled PL versus the longitudinal and transverse distances, respectively. (c) Normalized intensity of plasmon-coupled PL versus orientation of the SWNT (stars). The solid line is $\cos ^{2} \theta_{\text {SWNT }}$ fit.

$\left(\varepsilon_{1}=-22.5+i 1.4\right)$ and $\mathrm{SiO}_{x}\left(\varepsilon_{2}=2.25\right)$ at $\lambda=785 \mathrm{~nm}$. We calculated a plasmon decay length of $7.2 \mu \mathrm{m}$ for our configuration in accordance with the $L_{\mathrm{SPP}}$ obtained from the best-fit result of the experimental profile [solid line in Fig. 2(a)]. The PL intensity (stars) follows reasonably well the propagating plasmon profile confirming the plasmon excitation of the nanotube. The bump at $\sim 4.5 \mu \mathrm{m}$ in the longitudinal plasmon profile of Fig. 2(a) is not associated with the SPP scattering by the nanotube. The leakage radiation image of Fig. $1(\mathrm{~b})$ and its cross-section in Fig. 2(a) were measured on a long gold strip $(30 \mu \mathrm{m})$ to obtain a measurable $L_{\mathrm{SPP}}$ not affected by the strip termination. The origin of bump and oscillations in Fig. 2(a) are related to the plasmon excitation conditions, particularly angle and effective numerical aperture of the incident beam [20].

Figure 2(b) shows the normalized PL intensity plotted against the transverse plasmon profile. In our configuration, the SPP transverse cross-section is dictated by the geometry of the excitation spot. The transverse SPP intensity profile is approximated by a Gaussian shape $I_{T} \approx A \exp \left(-y^{2} / w\right)$, where $A$ and $w$ are fitting parameters and $y$ is the transverse distance. The intensity variation of the SWNT PL (stars) is reproducing the envelope of the SPP mode.

Surface plasmon interaction with the nanotubes provides higher oscillator strength than the one associated with standard PL [21]. An absolute value for the coupling efficiency of SPP to nanotube PL is difficult to access. However, we have shown above that the PL emission depends on the longitudinal and transverse profile of the propagating SPP. Any SWNT-based plasmonic device relying on this coupling scheme must take into account the intrinsic characteristics of the SPP longitudinal and transverse profiles. Damping of the electric field strength in propagating SPPs and the geometry of the excitation spot explains the weaker plasmon-exciton coupling beyond $\sim 7.2 \mu \mathrm{m}$ and $\sim 0.7 \mu \mathrm{m}$ in the longitudinal and transverse directions, respectively. 
Together, with SEM images, the longitudinal and transverse excitation efficiencies were used to deduce the orientation-dependent PL emission of the nanotube. The PL intensity with respect to nanotube orientation is plotted in Fig. 2(c). The intensity of the PL transition follows a $\cos ^{2} \theta_{\text {SWNT }}$ pattern, where $\theta_{\text {SWNT }}$ denotes the angle between the SWNT axis and $\boldsymbol{k}_{\mathrm{SPP}}$. The intensity is found to be maximal when the orientation of the nanotube is parallel to $\boldsymbol{k}_{\mathrm{SPP}}$ and minimal for the perpendicular orientation.

The interaction between excitonic states and propagating SPP modes results into SPP-exciton coupling in semiconducting SWNTs. The strongest optical response can be observed for light polarization along the nanotube axis $[19,22]$. The SPP-coupled PL transition in nanotubes is mediated via a dipole-dipole interaction between the dipole moment of the exciton transition and the electric field produced by the SPP. The physical phenomena of this interaction has been described by a system of twocoupled oscillators [23]. Since the SPP has a polarization component along its wave vector, excitons are efficiently excited when the nanotube axis is aligned in the SPP propagation direction. Thus, qualitatively the in-plane remote excitation of SWNT is the product of longitudinal, transverse, and orientation-dependent coupling efficiencies.

In summary, we presented a surface plasmon based excitation scheme for exciton generation in SWNTs. The coupling efficiency related to in-plane remote optical addressing of SWNTs by propagating SPPs has been interpreted in accordance to the SPP profile. Our results demonstrate that SWNTs can act as a sensor for propagating SPPs and provide a step towards the integration of plasmonic circuits based on SWNTs.

This work was funded by NanoSci E+ program under grant E2-PLAS (ANR-08-NSCI-007), the regional council of Burgundy program Plan Action Regional Innovation (PARI), the Deutsche Forschungsgemeinschaft (DFG) through HA4405/5-1 and the Nanosystems Initiative Munich (NIM).

\section{References}

1. A. M. Rao, E. Richter, S. Bandow, B. Chase, P. C. Eklund, K. A. Williams, S. Fang, K. R. Subbaswamy, M. Menon, A. Thess, R. E. Smalley, G. Dresselhaus, and M. S. Dresselhaus, Science 275, 187 (1997).
2. S. M. Bachilo, M. S. Strano, C. Kittrell, R. H. Hauge, R. E. Smalley, and R. B. Weisman, Science 298, 2361 (2002).

3. P. Avouris, M. Freitag, and V. Perebeinos, Nat. Photonics 2 , 341 (2008).

4. E. Gaufrès, N. Izard, A. Noury, X. L. Roux, G. Rasigade, A. Beck, and L. Vivien, ACS Nano 6, 3813 (2012).

5. M. Steiner, M. Freitag, V. Perebeinos, A. Naumov, J. P. Small, A. A. Bol, and P. Avouris, Nano Lett. 9, 3477 (2009).

6. M. Brun, A. Drezet, H. Mariette, N. Chevalier, J. C. Woehl, and S. Huant, Europhys. Lett. 64, 634 (2003).

7. Y. Wang, X. Liu, D. Whitmore, W. Xing, E. O. Potma, and A. Hartschuh, Opt. Express 19, 13454 (2011).

8. N. Hartmann, G. Piredda, J. Berthelot, G. Colas des Francs, A. Bouhelier, and A. Hartschuh, Nano Lett. 12177 (2012).

9. M. Böhmler, N. Hartmann, C. Georgi, F. Hennrich, A. A. Green, M. C. Hersam, and A. Hartschuh, Opt. Express 18 16443 (2010).

10. I. Pockrand, A. Brillante, and D. Mobius, J. Chem. Phys. 77, 6289 (1982).

11. G. P. Wiederrecht, J. E. Hall, and A. Bouhelier, Phys. Rev. Lett. 98, 083001 (2007).

12. E. Gaufres, N. Izard, X. L. Roux, D. Marris-Morini, S. Kazaoui, E. Cassan, and L. Vivien, Appl. Phys. Lett. 96, 231105 (2010).

13. K. Vasilev, W. Knoll, and M. J. Kreiter, J. Chem. Phys. 120, 3439 (2004).

14. A. Bouhelier and G. P. Wiederrecht, Opt. Lett. 30, 884 (2005).

15. S. G. Chou, F. Plentz, J. Jiang, R. Saito, D. Nezich, H. B. Ribeiro, A. Jorio, M. A. Pimenta, G. G. Samsonidze, A. P. Santos, M. Zheng, G. B. Onoa, E. D. Semke, G. Dresselhaus, and M. S. Dresselhaus, Phys. Rev. Lett. 94, 127402 (2005).

16. J. M. Marulanda, in Electronic Properties of Carbon Nanotubes, (InTech, 2011), Chap. 15.

17. C. D. Spataru, S. Ismail-Beigi, L. X. Benedict, and S. G. Louie, Phys. Rev. Lett. 92, 077402 (2004).

18. F. Wang, G. Dukovic, L. E. Brus, and T. F. Heinz, Science 308, 838 (2005).

19. A. Jorio, M. S. Dresselhaus, and G. Dresselhaus, Carbon Nanotubes (Springer, 2008).

20. J. C. Weeber, G. Colas des Francs, A. Bouhelier, and A. Dereux, Phys. Rev. B 83, 115433 (2011).

21. M. D. Leistikow, J. Johansen, A. J. Kettelarij, P. Lodahl, and W. L. Vos, Phys. Rev. B 79, 045301 (2009).

22. J. Lefebvre and P. Finnie, Phys. Rev. Lett. 98, 167406 (2007).

23. L. C. Andreani, G. Panzarini, and J. M. Gérard, Phys. Rev. B 60, 13276 (1999). 